\title{
AVALIAÇÃO DE PLANTAS COM ATIVIDADE DETERRENTE ALIMENTAR EM Spodoptera frugiperda (J .E.SMITH) E Anticarsia gemmatalis HUBNER
}

\author{
MARIA LUCIA SAITO * \\ ARNILDO POTT ** \\ JOSÉ MARIA GUSMAN FERRAZ *** \\ ROSELI DOS SANTOS NASCIMENTO ****
}

\begin{abstract}
Avaliou-se a atividade inibidora de alimentação de plantas nativas do Mato Grosso do Sul, utilizando lagartas dos insetos-pragas Spodoptera frugiperda e Anticarsia gemmatalis como organismos testes. Para os bioensaios foram preparados extratos alcoólicos pelo método da percolação, que após diluição foram aplicados em discos de folhas de milho ou de soja. Com os resultados das áreas consumidas foram calculados os índices de inibição alimentar. As espécies de plantas que apresentaram alguma atividade foram Agonandra brasiliensis, Ocotea suaveolens, Annona dióica e Machaerium hirtum (a mais ativa). Análises de grupos químicos presentes nas espécies mais ativas apontam a presença de saponinas, alcalóides, cumarinas ou flavonóides.
\end{abstract}

PALAVRAS-CHAVES: CONTROLE DE INSETOS; SUBSTÂNCIAS ATIVAS DE PLANTAS; DETERRENTE ALIMENTAR.

* Farmacêutica Bioquímica, Dra. em Química Orgânica, Fitoquímica, Embrapa Meio Ambiente, Jaguariúna, SP (e-mail: saito@cnpma.embrapa.br).

** Engenheiro Agrônomo, Dr. em Ciência das Pastagens, Embrapa Gado de Corte, Campo Grande, MS.

*** Biólogo, Dr. em Ecologia, Embrapa Meio Ambiente, Jaguariúna, SP.

**** Bacharel em Ciências Biológicas, Embrapa Meio Ambiente, Jaguariúna, SP. 


\section{INTRODUÇÃO}

O desenvolvimento da agricultura sustentável demanda métodos menos impactantes ao meio ambiente para o controle de pragas. Um dos métodos que têm mostrado muita utilidade é o controle que utiliza substâncias sintetizadas pelas plantas, cuja principal função parece ser a defesa das espécies vegetais contra insetos fitófagos. Essas substâncias secundárias defensivas podem pertencer à diversas categorias químicas (como terpenóides, alcalóides, glicósides, fenólicos, poliacetilenos, etc.) e exercem efeitos biológicos diversos sobre várias pragas (SINGH e WAHAB, 1998).

Dentre as substâncias úteis para o controle de insetos destacam-se as com ação inseticida, com ação esterilizadora, ou que apenas afastam os insetos das plantas como os repelentes e inibidores da alimentação. Em algumas situações (para a proteção da cultura) pode ser mais interessante o uso de substâncias que apenas desestimulem a ação dos herbívoros, pois a eliminação de alguns insetos pode causar desequilíbrio do sistema ecológico.

As plantas que produzem substâncias com ação inibidora da alimentação dos herbívoros, também conhecidas como deterrentes alimentares, vêm sendo estudadas há algumas dezenas de anos. GRAINGE e AHMED (1987) publicaram importante base de dados, com mais de 2400 espécies vegetais, centenas das quais com registro de atividade antialimentar para insetos. A espécie mais conhecida é a Azadirachta indica, pertencente à família das Meliáceas, e popularmente denominada de "Neem" ou "Nim". Muitos trabalhos relatam suas propriedades (MORDUE e BLACKWELL, 1993), havendo no comércio diversos produtos formulados com seu extrato. BHATHAL, DARSHAN e SINGH, (1994) experimentaram produto formulado com $80 \%$ de extrato de Azadirachta indica em larvas de terceiro instar de Spilosoma obliqua Walker. Observaram que a atividade antialimentar sobre o controle variou de $6,7 \%$ na menor concentração $(0,313 \%)$ até $86 \%$ na maior concentração $(5 \%)$. Como exemplo de outras espécies estudadas podem ser citados os deterrentes alimentares para insetos identificados em Tithonia diversifolia (Hemsl) Gray (tagitinin A, tagitinin C e hispidulin), que exibiram atividade dosedependente em larvas dos insetos Diacrisia obliqua [Spilarctia obliqua], Trabala vishnou e Epilachna vigintioctopunctata (DUTTA, CHAUDHURI, SHARMA, 1993). Já SZAFRANSKI, BLOSZYK e DROZDZ (1993) relatam atividade deterrente alimentar para adultos de Sitophilus granarius e Tribolium confusum, além da larva de Trogoderma granarium e T. confusum nas espécies africanas das famílias Meliaceae, Asteraceae e Simaroubaceae. Muitos outros exemplos de plantas com atividade antialimentar podem ser verificados na literatura. JAIN e TRIPATHI (1993) descreveram aspectos biológicos e químicos das substâncias inibidoras de alimentação presentes em plantas que poderiam ser utilizadas para o manejo de pragas da agricultura. Relacionaram também, diversas classes de substâncias já isoladas de algumas espécies vegetais com atividade inibidora da alimentação de insetos.

No presente trabalho foi estudada a atividade deterrente alimentar de algumas espécies de plantas brasileiras, utilizando Spodoptera frugiperda e Anticarsia gemmatalis, lagartas do cartucho de milho e da soja, respectivamente, como insetos-testes. Pouquíssimas informações foram obtidas sobre as espécies botânicas escolhidas para este trabalho, tanto sob os pontos de vista químico como farmacológico ou biológico, não sendo encontrada na literatura pesquisa com enfoque em controle de pragas agrícolas.

\section{MATERIAL E MÉTODOS}

Os materiais botânicos (Tabela 1) foram coletados na região de Nhecolândia, no Mato Grosso do Sul. Após identificados, os exemplares foram depositados no Herbário da Embrapa Pantanal (CPAP). 


\section{TABELA 1 - ESPÉCIES DE PLANTAS UTILIZADAS NOS TESTES DE DETERRÊNCIA E DE} TOXICIDADE A INSETOS

\begin{tabular}{|c|c|c|}
\hline EspØcie e parte usada & Fam lia & Nome comum \\
\hline Agonandra brasiliensis Miers raiz e folha & Opiliaceae & Tinge-cuia \\
\hline Alchornea discolor Poepp. folha & Euphorbiaceae & Uva-brava \\
\hline Annona cornifolia St. Hil. folha & Annonaceae & Ata-de-cobra \\
\hline Annona dioica St. Hil. folha e caule & Annonaceae & Ariticum \\
\hline Bunchosia paraguariensis Nied folha e caule & Malpighiaceae & - \\
\hline Copaifera martii Hayne folha & Leguminosae & Guaranazinho \\
\hline Curatella americana $\mathrm{L}$. folha & Dilleniaceae & Lixeira \\
\hline \multicolumn{3}{|l|}{ Davilla elliptica St.Hil. folha e caule } \\
\hline \multicolumn{3}{|l|}{ Erytroxilulm anguifugum Mart. caule } \\
\hline Fagara hassleriana Chod. folha e caule & Rutaceae & Maminha \\
\hline Licania parvifolia Huber folha & Chrysobalanaceae & Pimenteira \\
\hline Machaerium hirtum (Vell.) Stellf. caule & Leguminosae & Barreiro \\
\hline Ocotea suaveolens Hassl. folha & Lauraceae & Caneleira \\
\hline Rhamnidium elaeocarpum Reiss. folha & Rhamnaceae & Cabrito \\
\hline Richardia grandiflora (C.et S.) parte ađrea & Rubiaceae & Ipeca \\
\hline Sebastiania hispida (Mart.) Pax caule & Euphorbiaceae & Mercœio \\
\hline Tocoyena formosa (C.etS.) Schum. caule & Rubiaceae & Olho de boi \\
\hline Unonopsis lindmanii Fries - caule & Annonaceae & Pindaiva preta \\
\hline
\end{tabular}

Foram utilizadas (como insetos-testes) lagartas de Spodoptera frugiperda (J.E. Smith, 1797) e de Anticarsia gemmatalis Hubner, 1818, mantidas em dieta artificial no Laboratório de Entomologia da Embrapa Meio Ambiente.

Os extratos das plantas foram obtidos pelo método de percolação, utilizando-se etanol $94^{\circ} \mathrm{GL}$ como líquido extrator. Eliminou-se o solvente totalmente por destilação em rotaevaporador, a $\pm 55^{\circ} \mathrm{C}$. $\mathrm{O}$ resíduo foi ressuspenso em água ou solução aquosa com 10\% do tensoativo Tween quando havia dificuldade na dissolução. Ajustou-se a concentração final para corresponder à da planta original, ou seja, $1 \mathrm{~g}$ de planta $=1 \mathrm{~mL}$ de extrato final.

Para o teste de deterrência usaram-se lagartas no $5^{\circ}$ instar, sendo a unidade experimental constituída de 40 a 50 placas de Petri ( $10 \mathrm{~cm}$ de diâmetro). Colocou-se uma lagarta em cada placa e 6 discos de aproximadamente $1 \mathrm{~cm}$ de diâmetro de folhas de milho ou soja de acordo com a espécie de inseto, intercalando-se discos tratados e não-tratados com o extrato (Figura 1). O procedimento foi repetido para cada extrato e para o solvente utilizado para dissolver os extratos. Após duas horas, os discos foram retirados e sua área foliar medida para a verificação do consumo em aparelho marca Licor, com precisão de $0,01 \mathrm{~cm}^{2}$. Para a análise comparativa dos dados foi utilizado o índice de inibição alimentar, baseado na seguinte equação (SIMMONDS et al., 1987):

$$
I=(A c-A t) /(A c+A t)
$$

na qual:

$\mathrm{Ac}=$ Área consumida do disco controle $\left(\mathrm{mm}^{2}\right)$.

At $=$ Área consumida do disco tratado $\left(\mathrm{mm}^{2}\right)$. 
No caso de resultado positivo, o disco com a amostra deve ser menos consumido que o disco controle. Valores acima de 0,50 são considerados índices significativos de inibição alimentar e índices acima de 0,90 são altamente eficientes como deterrente alimentar (SIMMONDS et al., 1987). Para efeito comparativo foram preparados extratos de Melia azedarach, acatada como deterrente alimentar para vários insetos (GRAINGE E AHMED, 1987). Considerou-se a média dos índices encontrados na repetição dos ensaios.

\section{FIGURA 1 - TESTE PARA AVALIAR A ATIVIDADE DETERRENTE ALIMENTAR DE Spodoptera frugiperda}

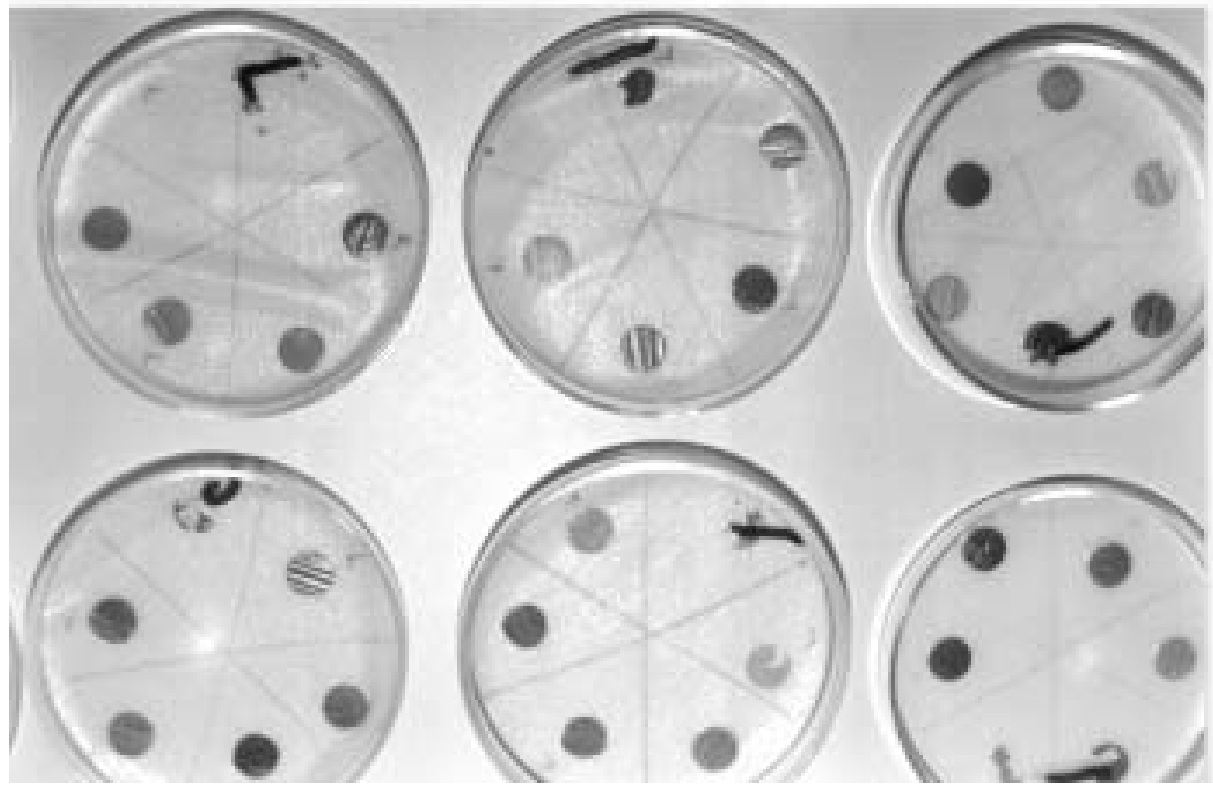

Complementou-se o teste de atividade deterrente alimentar de extrato de Machaerium com dieta artificial, preparada pela adição de 2,5 e de $5 \%$ de extratos. Empregaram-se 50 lagartas do 1 o instar e 50 do $2^{\circ}$ instar de Spodoptera frugiperda, sendo cada grupo alimentado com dietas nas duas concentrações mencionadas até a fase de pupa.

Para a triagem química dos grupos de substâncias foram utilizadas as metodologias citadas por MATOS (1988), que consistem em reações químicas com reagentes específicos para os grupos funcionais presentes.

\section{RESULTADOS E DISCUSSÃO}

Quatro dos extratos apresentaram alguma atividade antialimentar (Tabela 2), ou seja, os de folhas de Agonandra brasiliensis e Ocotea suaveolens, fruto de Annona dióica e casca de Machaerium hirtum. Os índices de inibição apresentados por essas espécies mostraram-se menores que o da espécie-referência (Melia azedarach). Atribuiu-se tal fato às prováveis diferenças na concentração de princípios ativos presentes nos extratos das plantas. Verificou-se maior constância nos resultados e atividades deterrente e inseticida significativas nas repetições efetuadas com a espécie Machaerium hirtum. Resolveu-se, então, avaliar melhor seu extrato para as lagartas dos insetos Spodoptera frugiperda e Anticarsia gemmatalis. 
TABELA 2 - RESULTADO DO TESTE DE DETERRÊNCIA

\begin{tabular}{lcc}
\hline EspØcie & Parte da planta usada & $\begin{array}{c}\text { "ndice de } \\
\text { deterrcrcia }\end{array}$ \\
\hline Agonandra brasiliensis & Raiz & $-0,09$ \\
Agonandra brasiliensis & Folha & $\mathbf{0 , 3 0}$ \\
Alchornea discolor & Folha & $-0,17$ \\
Annona cornifolia & Folha & 0,08 \\
Annona dioica & Fruto & $\mathbf{0 , 3 3}$ \\
Bunchosia paraguariensis & Folha & $-0,04$ \\
Bunchosia paraguariensis & Caule & $-0,37$ \\
Copaifera martii & Folha & $-0,05$ \\
Curatella americana & Folha & $-0,11$ \\
Davilla elliptica & Caule & 0,12 \\
Davilla elliptica & Folha & 0,14 \\
Erytroxilum anguifugum & Caule & $-0,30$ \\
Fagara hassleriana & Caule & 0,22 \\
Fagara hassleriana & Folha & 0,22 \\
Licania parvifolia & Folha & $-0,06$ \\
Licania parvifolia & Caule & 0,15 \\
Machaerium hirtum & Caule & $\mathbf{0 , 3 3}$ \\
Ocotea suaveolens & Caule & 0,01 \\
Ocotea suaveolens & Folha & $\mathbf{0 , 2 0}$ \\
Rhamnidium elaeocarpum & Folha & 0,05 \\
Richardia grandiflora & Parte aØrea & 0,08 \\
Sebastiania hispida & Caule & 0,10 \\
Tocoyena formosa & Caule & $-0,23$ \\
Unonopsis lindmanii & Caule & 0,08 \\
Unonopsis lindmanii & Folha & 0,16 \\
Melia azedarach & fruto & $\mathbf{0 , 5 0}$ \\
\hline & & \\
\hline
\end{tabular}

Índice de deterrência $=(\mathrm{Ac}-\mathrm{At}) /(\mathrm{Ac}+\mathrm{At})$. Varia de -1 a 1.

$A c=$ média da área consumida do disco controle $\left(\mathrm{mm}^{2}\right)$.

$A t=$ média da área consumida do disco contendo a amostra $\left(\mathrm{mm}^{2}\right)$.

Para observar a correspondência dose-resposta foram avaliadas diversas concentrações dos extratos, juntamente com o extrato de frutos de Melia azedarach. A curva dose-resposta não foi perfeita, mas apresentou quase sempre melhores resultados nas maiores concentrações (Tabela $3 \mathrm{e}$ Figura 2). Esse tipo de desvio pode ocorrer com certa freqüência em princípios ativos obtidos de plantas, conforme relatado por SIMMONDS (2001) para exemplificar o comportamento de insetos 
frente ao flavonóide rutina. Em concentração maior que $10^{-3} \mathrm{M}$, essa substância atua como inibidor da alimentação de Heliothis zea e de Helicoverpa armigera nos estádios finais de desenvolvimento. Já em concentração menor que $10^{-4}$ atua como fagoestimulante para essas mesmas lagartas. Deve-se lembrar que lagartas com estádios diferentes de desenvolvimento também podem apresentar resultados diferentes.

Nesse ensaio, a atividade dos extratos de Machaerium mostrou-se menor que a do extrato dos frutos de Melia em concentrações equivalentes. Experimentos com diversos solventes extratores evidenciaram que a atividade concentra-se na fração mais polar. Porém, tanto a fração etanólica como a de acetato de etila apresentaram atividade. Quando se fracionou o extrato etanólico com vistas ao isolamento do princípio ativo verificou-se perda de atividade.

TABELA 3 - AVALIAÇÃO DA ATIVIDADE DETERRENTE ALIMENTAR PARA INSETOS DOS EXTRATOS DA CASCA DE Machaerium hirtum E Melia azedarach, EM 5 CONCENTRAÇÕES DIFERENTES

\begin{tabular}{c|cc}
\hline Concentra ${ }^{\circ} 0$ & M.hirtum & Melia \\
& I & I \\
\hline 2,5 & 0,41 & 0,96 \\
2,0 & 0,38 & 0,68 \\
1,5 & $-0,10$ & 0,55 \\
1,0 & $-0,25$ & 0,66 \\
0,5 & 0,01 & 0,36 \\
\hline
\end{tabular}

I = índice de deterrência alimentar.

FIGURA 2 - ATIVIDADE ANTIALIMENTAR DOS EXTRATOS ETANÓLICOS DAS ESPÉCIES Melia azedarach e Machaerium hirtum

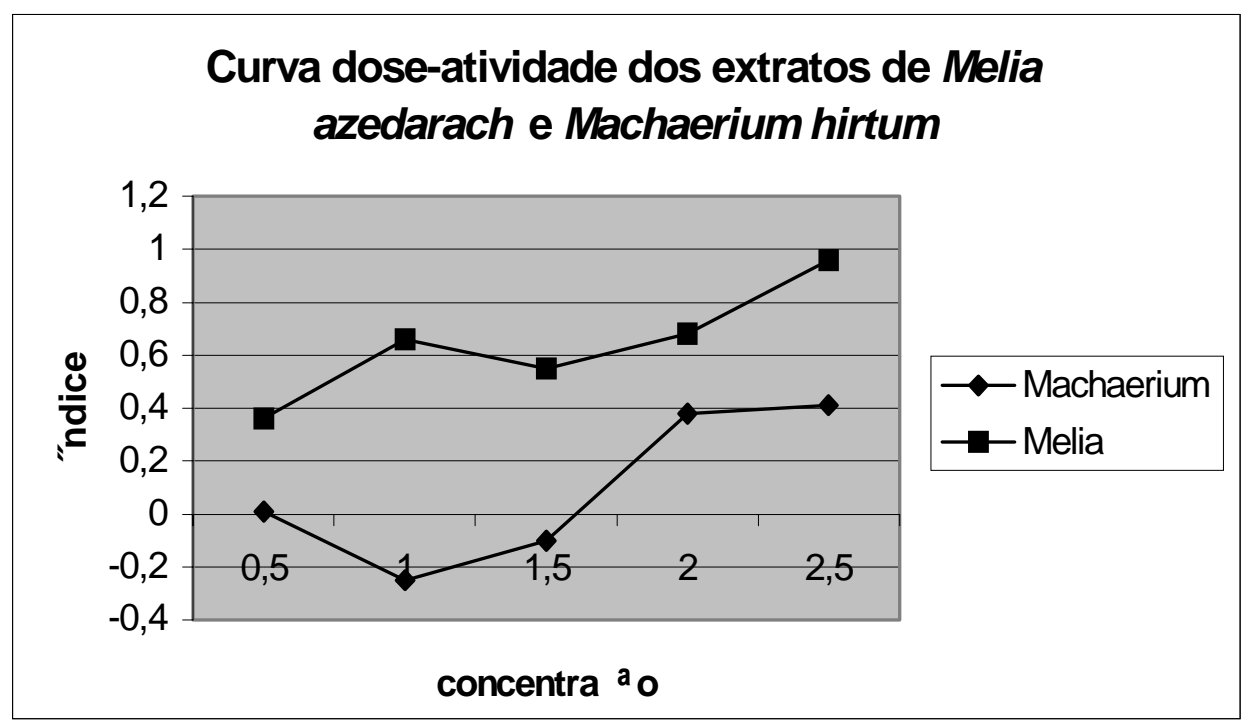

Observou-se elevada mortalidade quando foi adicionado extrato de Machaerium nas dietas das lagartas. As lagartas de $2^{\circ}$ instar mostraram-se as mais sensíveis com mortalidade de $85 \%$ na 
concentração de 2,5\% e de 100\% na concentração de 5\%. As lagartas de $1{ }^{\circ}$ instar apresentaram mortalidade de $48 \%$ e $63 \%$ na concentração de 2,5 e $5 \%$, respectivamente, atribuindo-se a mortalidade à não-ingestão de alimentos (Figura 3 e 4). A menor mortalidade em lagartas de $1^{\circ}$ instar talvez indique maior facilidade de adaptação nessa fase de desenvolvimento, mas essa hipótese teria que ser avaliada.

FIGURA 3 - TESTE DE TOXICIDADE EM Spodoptera frugiperda DO $2^{\circ}$ INSTAR COM EXTRATO DE Machaerium (2,5\%) AVALIADO APÓS 13 DIAS

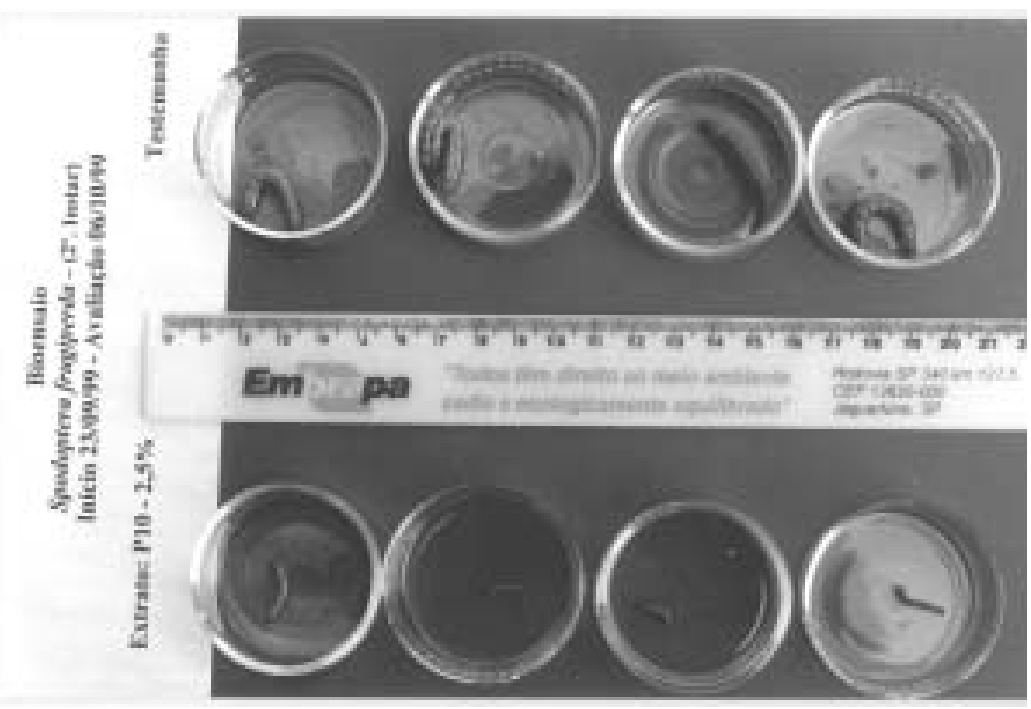

FIGURA 4 - TESTE DE TOXICIDADE EM Spodoptera frugiperda DE $2^{\circ}$ INSTAR COM EXTRATO DE Machaerium (5,0\%) AVALIADO APÓS 13 DIAS

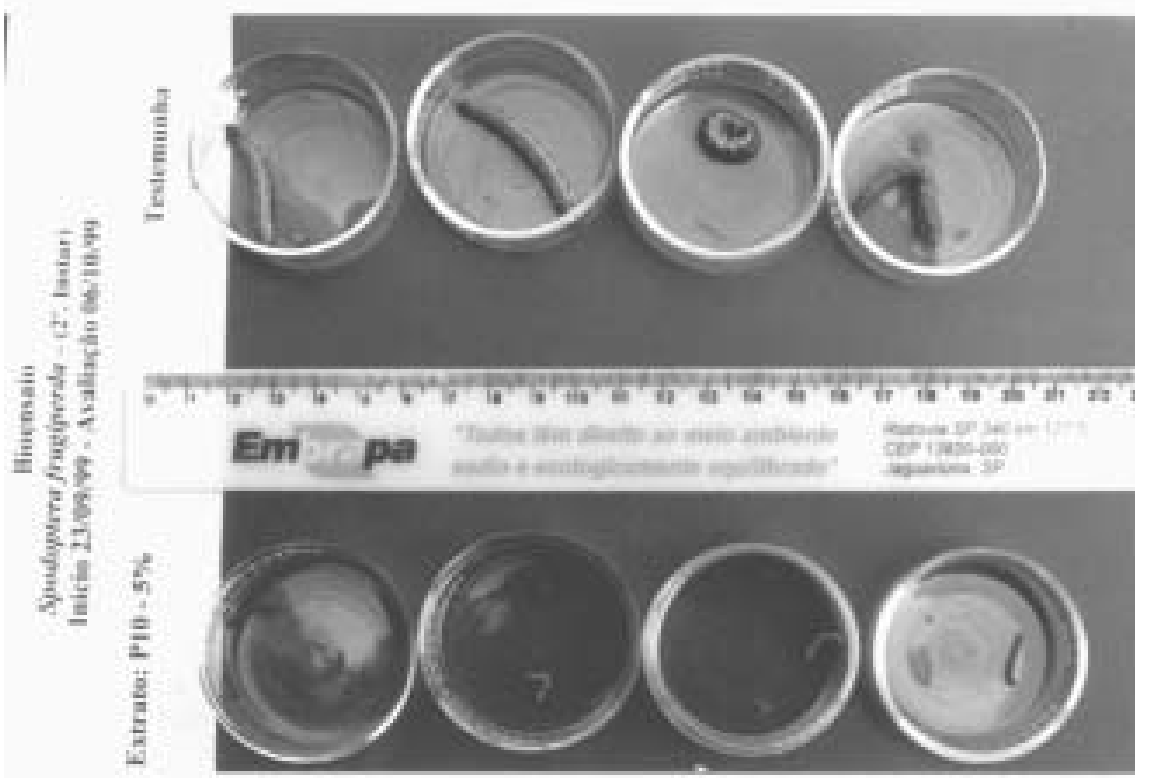

Os resultados das triagens químicas (em todas as amostras) para avaliar a presença ou ausência dos principais grupos de substâncias que comumente apresentam atividade biológica constam da Tabela 4.

As espécies que apresentaram atividade inibidora de alimentação contém, principalmente, saponinas, alcalóides, flavonóides e cumarinas. Tais classes de substâncias figuram freqüentemente 
como responsáveis por essa atividade em diversas espécies de plantas, como indica a extensa lista bibliográfica sobre o assunto (MURTHY et al., 1998; PRASAD et al., 1998; CALCAGNO et al., 2002; MORIMOTO et al., 2002; PIUBELLI et al., 2003; SHARMA e NORRIS, 1994; JAIN e TRIPATHI, 1991; RITU et al., 1999; WALIGORA, 1998; PARK et al., 2000). Informações sobre as classes de substâncias que estão presentes nas espécies de plantas ativas são de grande importância para se iniciar estudos fitoquímicos, de isolamento e identificação dos princípios ativos de cada planta.

Algumas espécies coletadas em épocas distintas apresentaram composição diferente, o que reforça a necessidade de melhor estudo da química dessas plantas para viabilizar sua utilização na prática (Tabela 4).

\section{TABELA 4 - TRIAGEM QUÍMICA DOS GRUPOS DE SUBSTÂNCIAS PRESENTES}

\begin{tabular}{|c|c|c|c|c|c|c|c|}
\hline \multirow[t]{2}{*}{ EspØcies } & \multirow[t]{2}{*}{$S p$} & & a v & \multirow[t]{2}{*}{ Tan } & \multirow[t]{2}{*}{ Alc } & Antraq & \multirow{2}{*}{$\begin{array}{c}\text { Cumar } \\
\text { Livres Comb }\end{array}$} \\
\hline & & Al & $\mathrm{Mg}$ & & & Livres Comb & \\
\hline
\end{tabular}

\begin{tabular}{|c|c|c|c|c|c|c|c|c|c|}
\hline Agonandra-Ra & + & - & - & - & - & - & - & + & + \\
\hline Agonandra-Fo & + & + & - & - & - & - & - & + & - \\
\hline Alchornea-Fo & - & - & - & + & - & - & - & - & - \\
\hline Annona cornifolia - $\mathrm{Ca}$ & - & - & 0 & + & - & - & - & + & - \\
\hline Annona cornifolia - Fo & + & + & 0 & - & - & - & - & - & - \\
\hline Annona cornifolia $\mathbf{b}-\mathrm{Ca}$ & + & - & - & - & - & - & - & + & + \\
\hline Annona cornifolia b - Fo & + & + & - & - & + & - & - & + & + \\
\hline Annona dioica-Fr & - & - & - & - & + & - & - & + & - \\
\hline Bunchosia-Ca & - & + & - & - & - & - & - & + & + \\
\hline Bunchosia-Fo & - & + & - & - & - & - & - & + & + \\
\hline Copaifera-Fo & - & - & - & + & - & + & - & - & - \\
\hline Curatella-Ca & - & - & - & + & - & + & - & - & - \\
\hline Curatella-Fo & - & + & - & - & - & - & - & - & - \\
\hline Davilla Fo & + & - & + & - & - & - & - & - & - \\
\hline Davilla $\mathrm{Ca}$ & + & - & + & - & - & - & - & - & - \\
\hline Fagara-Ca & - & + & - & + & - & - & - & + & + \\
\hline Fagara-Fo & - & - & - & + & - & - & - & - & - \\
\hline Fagara $\mathbf{b}-\mathrm{C}$ a & - & + & - & - & - & - & - & + & - \\
\hline Licania-Ca & - & - & - & - & - & + & - & - & - \\
\hline Licania-Fo & + & + & + & + & - & - & - & - & - \\
\hline Machaerium-Cas & + & - & - & - & - & + & - & - & + \\
\hline Ocotea - Fo & - & + & - & - & + & - & - & - & + \\
\hline Ocotea-Ca & + & - & - & - & + & - & - & + & + \\
\hline Rham nidium - Fo & - & + & + & - & - & + & - & + & + \\
\hline Richardia $-\mathrm{Pa}$ & - & + & + & - & - & - & - & - & - \\
\hline Richardia-Ra & - & + & 0 & + & - & - & - & + & + \\
\hline Sebastiania-Ca & - & + & - & + & - & - & - & - & - \\
\hline Sebastiania-Fo & - & + & - & + & - & - & - & - & - \\
\hline Sebastiania b-Ca & - & & - & + & - & & - & + & - \\
\hline Sebastiania b-Fo & - & & - & + & - & - & - & - & - \\
\hline Tocoyena-Fo & + & + & - & - & - & - & - & - & - \\
\hline Tocoyena-Fr & + & + & - & - & - & - & - & + & - \\
\hline Tocoyena-Ca & + & + & - & + & - & - & - & + & - \\
\hline Unonopsis - Fo & + & + & 0 & - & - & - & - & - & - \\
\hline Unonopsis-Ca & - & - & 0 & + & - & + & - & + & - \\
\hline
\end{tabular}

$\mathrm{Sp}=$ saponinas; Flav = flavonóides $(\mathrm{Mg}=$ teste com magnésio $) ;$ Alc = alcalóides; Tan = taninos; Antraq = antraquinonas; Cumar = cumarinas; Fo = folha; $\mathrm{Ca}=$ caule; $\mathrm{Pa}=$ parte aérea; $\mathrm{Ra}=$ raiz; $\mathrm{Fr}=$ fruto; Cas = casca do caule. Sinal + (presença), - (ausência) e 0 (não realizado).

Os nomes seguidos pela letra $\mathbf{b}$ referem-se a segunda coleta da mesma espécie, com resultados diferentes. 


\section{CONCLUSÃO}

Os estudos realizados confirmam o potencial da flora brasileira para obtenção de materiais menos poluentes para o controle de pragas agrícolas. Os ensaios biológicos indicaram atividade antialimentar em pelo menos 3 das 18 espécies estudadas. Incertezas quanto ao tipo e concentração do princípio ativo presente nessas espécies evidenciam a necessidade de estudos mais aprofundados quanto aos componentes químicos responsáveis por essa atividade.

Variações na composição química das plantas foi observada quando a mesma espécie foi coletada em diferentes épocas. A ocorrência dessa variação é muito comum em plantas, podendo influir nos resultados dos bioensaios e exigindo cuidado nas avaliações.

Das espécies estudadas, a Leguminosa Machaerium demonstrou maior potencial como inibidora de alimentação de Spodoptera frugiperda e Anticarsia gemmatalis, sendo o componente químico ativo observado nas frações polares do extrato. Os resultados dos testes químicos indicaram a presença de saponinas e atraquinonas nessa espécie. É possível que a responsável pela atividade inibidora seja a saponina, mas estudos químicos e biológicos adicionais terão que ser conduzidos para avaliação da utilidade dessa espécie.

\section{ABSTRACT}

EVALUATION OF PLANTS WITH ANTIFEEDANT ACTIVITY IN Spodoptera frugiperda (J.E. SMITH) AND Anticarsia gemmatalis HUBNER

The antifeedant activity of native plants from Mato Grosso do Sul against caterpillars and phytophagous insects Spodoptera frugiperda and Anticarsia gemmatalis with test organisms was evaluated. For the bioassays alcoholic extracts were prepared by the percolation method which after dilution where applied on discs of corn and soya leaves. With the results of consumed areas the antifeeding index were calculated. The plant species that presented some activity were Agonandra brasiliensis, Ocotea suaveolens, Annona dioica and Machaerium hirtum (the most active). Chemical group analysis presented in the most active species revealed the presence of saponins, alkaloids, cumarins or flavonoids .

KEY-WORDS: INSECT CONTROL; PLANT ACTIVE COMPOUNDS; ANTIFEEDANT.

\section{REFERÊNCIAS}

1 BHATHAL, S.S.; DARSHAN, S.; SINGH, D. Feeding deterrency of Neemark against third instar larvae of hairy caterpillar, Spilosoma obliqua Walker. Journal of Entomological Research, v.18, n.4, p.387-389, 1994.

2 CALCAGNO, M.P.; COLL, J.; LLORIA, J.; FAINI, F.; ALONSO, A. M.E. Evaluation of synergism in the feeding deterrence of some furanocoumarins on Spodoptera littoralis. Journal of Chemical Ecology, v. 28, n. 1, p.175-191, 2002.

3 DUTTA, P.; CHAUDHURI, R.P.; SHARMA, R.P. Insect feeding deterrents from Tithonia diversifolia (Hemsl) Gray. Journal of Environmental Biology, v.14, n. 1, p. 27-33, 1993.

4 GRAINGE, M.; AHMED, S. Handbook of plants with pest-control properties. New York: Wiley-Interscience, 1987. $470 \mathrm{p}$.

5 JAIN, D.C.; TRIPATHI, A. K. Potential of natural products as insect antifeedants. Phytotherapy Research, v. 7, p. $327-334,1993$.

6 JAIN, D.C.; TRIPATHI, A.K. Insect feeding-deterrent activity of some saponin glycosides. Phytotherapy Research, v. 5 , n. 3, p. 139-141, 1991.

7 MATOS, F.J.A. Introdução à fitoquímica experimental. Fortaleza: UFC, 1988. 128 p.

8 MORDUE, A.J.; BLACKWELL, A. Azadirachtin: an update. Journal of Insect Physiology, v.39, n.11, p.903-924, 1993.

9 MORIMOTO, M.; TANIMOTO, K.; SAKATANI, A.; KOMAI, K. Antifeedant activity of an anthraquinone aldehyde in Galium aparine L. against Spodoptera litura F. Phytochemistry, v. 60, n.2, p.163-166, 2002.

10 MURTHY, S.S.; AMARJIT, K.; SREENIVASULU, B.; SARMA, P.N.; AMARJIT, K. Antifeedant activity of some new Schiff's bases and dicoumarins against the larvae of Spodoptera litura $F$. Indian Journal of Experimental Biology, v., 36, n. 7, p. 724-727, 1998. 
11 PARK, I.K.; LEE, H.S.; LEE, S.G.; PARK, J.D.; AHN, Y.J. Antifeeding activity of isoquinoline alkaloids identified in Coptis japonica roots against Hyphantria cunea (Lepidoptera: Arctiidae) and Agelastica coerulea (Coleoptera: Galerucinae). Journal of Economic Entomology, v. 93, n. 2, p.331-335, 2000.

12 PIUBELLI, G.C.; HOFFMANN, C. C.B.; ARRUDA, I.C.; FRANCHINI, J.C.; LARA, F.M. Flavonoid increase in soybean as a response to Nezara viridula injury and its effect on insect-feeding preference. Journal of Chemical Ecology, v. 29 , n. 5 , p. $1223-1233,2003$.

13 PRASAD, C.V.S.S.; DEEPIKA, S.; RAM, G.M.; SREENIVASULU, B.; SARASWAT, D. Effect of certain distyryl coumarins against the larvae of Spodoptera litura (Fabricius). Indian Journal of Plant Protection, v. 26, n.1, p. 75-77, 1998.

14 RITU, S.; SHUKLA, Y.N.; SUSHIL, K.; SRIVASTAVA, R.; KUMAR, S. Recent advances in the chemistry of insect antifeedants. Journal of Medicinal and Aromatic Plant Sciences, v. 21, n. 1, p. 59-76, 1999.

15 SHARMA, H.C.; NORRIS, D.M. Biochemical mechanisms of resistance to insects in soybean: extraction and fractionation of antifeedants. Insect Science and its Application, v. 15, n. 1, p. 31-38, 1994.

16 SIMMONDS, M.S.J. Importance of flavonoids in insect-plant interactions: feeding and oviposition. Phytochemistry, v. 56, n. 3, p. 245-252, 2001.

17 SIMMONDS, M.S.J.; BLANEY, W.M.; LEY, S.V.; SAVONA, G.; BRUNO, M.; RODRIGUEZ, B. The antifeedant activity of clerodane diterpenoids from Teucrium. Phytochemistry, v. 28, n. 4, p. 1069-1071, 1989.

18 SINGH, R.P.; SINGH, S; WAHAB, S.; DHALIWAL, G.S.; ARORA, R.; RANDHAWA, N.S.; DHAWAN, A.K. Biodiversity and importance of botanical pesticides. In: DHALIWAL, G.S.; ARORA, R.; RANDHAWA, N.S. (Ed.). Ecological agriculture and sustainable development. Chandigarh: Centre for Research in Rural and Industrial Development, 1998. v.2, p. 128-145.

19 SZAFRANSKI, F; BLOSZYK, E; DROZDZ, B. Deterrent activity of African plant extracts against selected stored product insect pests. Acta Horticulturae, n.331, p.319-322, 1993.

20 WALIGORA D. Biological activity of secondary plant substances glucosinolates, alkaloids and saponins, expressed by their effects on development of Colorado potato beetle, Leptinotarsa decemlineata say. Journal of Plant Protection Research, v.38, n. 2, p.158-173, 1998. 